\title{
Honeycomb Structure and Key Technologies of 5G Wireless Communication Network
}

\author{
Siyan Duan \\ WenjingCollege .Yantai universityInformation. Engineering Department \\ 264005, China
}

\begin{abstract}
In recent years, wireless technology in China has been further improved. Wireless system designers have also begun research on $5 \mathrm{G}$ wireless communication systems and obtained good research results. The application of the $5 \mathrm{G}$ cellular structure can effectively solve the problems existing in the current wireless technology application, thereby promoting the further improvement of the wireless technology level. This paper mainly discusses the cellular technology and the key technologies of 5G wireless communication network.
\end{abstract}

\section{Introduction}

As people's access to information and speed requirements are getting higher, the current development of mobile communications is also facing great challenges. Therefore, relevant researchers need to further strengthen the research on $5 \mathrm{G}$ wireless communication technology to meet the growing communication needs of people.

\section{5G Wireless Cellular Technology}

In recent years, China's communications industry has developed rapidly, and higher requirements are placed on the speed and bandwidth of wireless communications. However, most users use wireless devices indoors, but no matter the users stay indoor or outdoor, the current traditional cell structure is mostly an outdoor base station in the middle of the mobile communication cell. In the process of wireless communication, the users' signals also need to pass through the wall of the building, thereby generating a large amount of penetration. Losses can also have a very large impact on data transmission rate, spectral efficiency, and energy efficiency. The design idea of $5 \mathrm{G}$ wireless cellular technology is micro base station, and its principle is basically similar to that of WIFI used in China. In this technology, the mode of small base station arrangement in indoors and outdoors is used to avoid the signal generated during the wall penetration process. Generally, the multi-antenna technology and the distributed antenna technology are mainly used in the outdoor, and the external base station is mainly composed of a plurality of antenna arrays, and can be connected to the BS unit through the mode of the optical fiber connection. When users are outdoors, the number of antennas they hold is limited, but the communication between the brothers' antennas can also form an antenna network and can form a communication link with the real MOMO.

It can be said that the key idea of $5 \mathrm{G}$ honeycomb structure design is mainly to divide the indoor and outdoor scenes, the purpose of which is to avoid the penetration loss of the building wall to the signal. With the aid of distributed antenna systems and massive MIMO technology, it is possible to deploy dozens or even hundreds of distributed antenna arrays in the same base station. Current MIMO systems can utilize a large number of antenna arrays to achieve greater potential capacity gain. The antenna array provided in the cell can be connected to the BS by means of an optical fiber through the application of the outdoor base station, and can form a virtual large-array antenna on the basis of the above. An external base station or a BS distributed antenna array will be installed outside each building, and the large antenna array can be directly connected to the wireless access point and the indoor user communication facility in the building through the cable, which can be used in the short-term operation. The infrastructure cost is improved to a certain extent, but in the long-term operation, the average throughput of the cell and the spectrum efficiency are greatly improved. The cell under 5G communication technology should also be heterogeneously processed with macrocell and microcellular technology. In order to adapt to high mobility users such as users in vehicles and high-speed trains, the concept based on mobile femtocell is also proposed. The large antenna array is located outside the vehicle and can communicate with the indoor BSS, and a mfemtocell and related users are regarded by the BS as a single unit, and can effectively reduce the signaling overhead and promote the data rate service quality to be further improved and upgrade [1]. Figure 1 shows the $5 \mathrm{G}$ heterogeneous cellular architecture. 


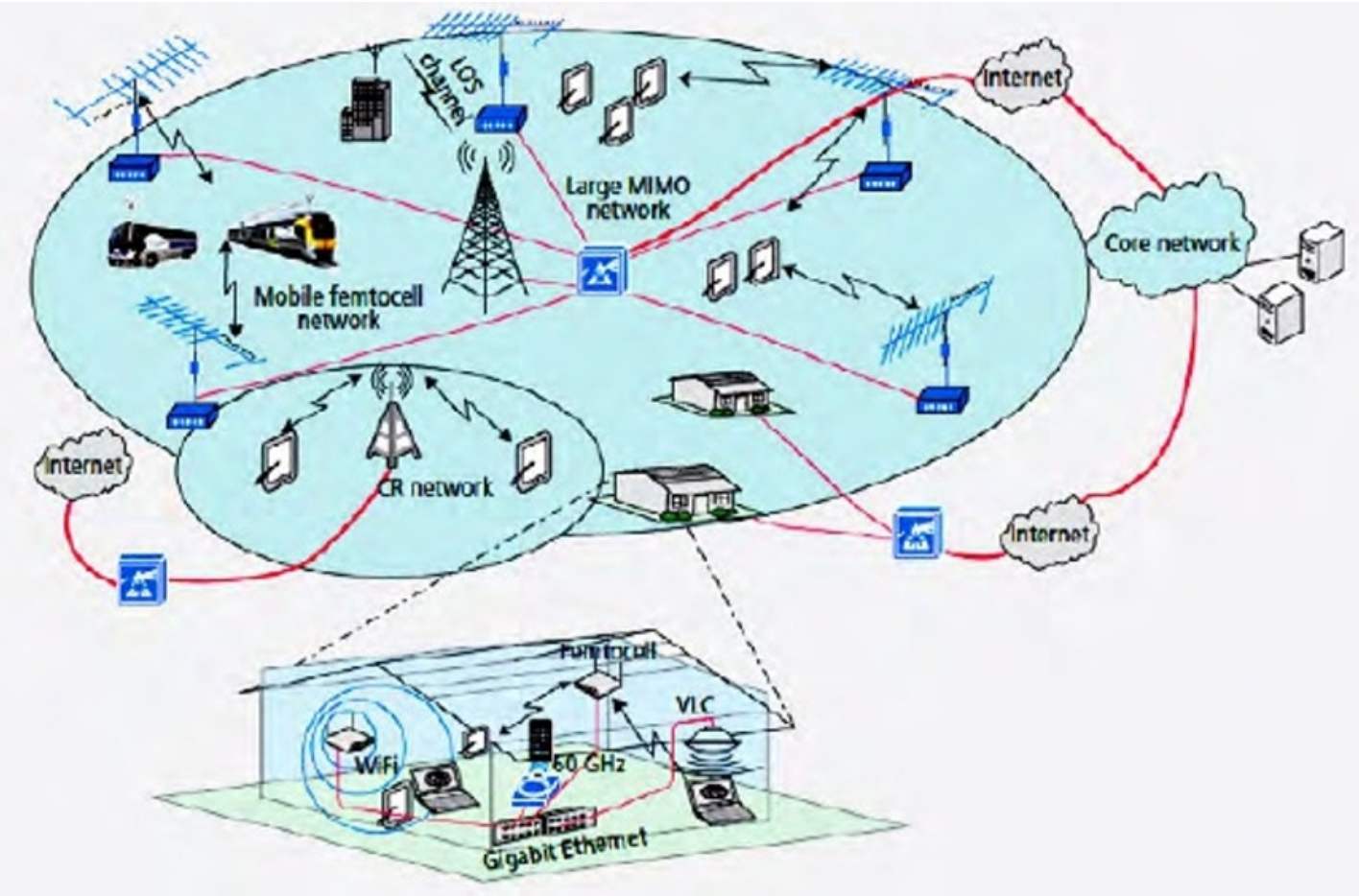

Figure 1: Wireless heterogeneous cellular structure

\section{Analysis of the Application Advantages of 5G Wireless Cellular Structure}

\subsection{High Frequency Data Gives Users a Better Experience}

In the daily use of wireless communication networks, people mostly use them in indoor environments. However, the traditional living environment is mostly to establish outdoor base stations in the middle of the cells used for communication, and will also be subject to construction during communication and the influence of the walls. The $5 \mathrm{G}$ cellular architecture is built on the basis of $4 \mathrm{G}$ networks. It is mainly for the network with large traffic and dense coverage, and then the transmission base station is used to realize the effective transmission of various data. The speed of information transmission is further accelerated and the frequency of transmission traffic can be increased. In addition, in the design process of $5 \mathrm{G}$ cellular structure, it is necessary to stand on the user's standpoint to think about the problem, in order to meet the requirements of users for the speed of data traffic transmission, and to further enhance the user's traffic experience [2].

\subsection{Ultra Short Delay}

In order to obtain an efficient information transmission effect, it is necessary to fully pay attention to the transmission time consumption. The application of the $5 \mathrm{G}$ cellular structure can give the $5 \mathrm{G}$ network a technical means to be more in line with the system operation in the hardware, and can also be carried out through the careful division of physical layer transmission route which can greatly reduce the number of repetitions in the transmission process, thereby improving the efficiency of information transmission.

\subsection{Massive Terminal Technology}

With the extension of time, the capacity of $5 \mathrm{G}$ network will be greatly improved. In order to meet the various requirements of high-frequency data traffic connection, it is necessary to apply networking technology to reduce the burden of the base point. It can avoid the loss of data when the outdoor base station is transmitted indoors, and can further increase the efficiency of delivery, and provide users with hierarchical and differentiated services.

\section{Brief Analysis of Key Technologies of 5G Wireless Communication Network}

Under the characteristics of cellular structure, 5G wireless network is inseparable from many key technologies. The development prospects of technologies such as large number of MIMO, new mobile base stations and green communication will directly affect the performance changes of $5 \mathrm{G}$ wireless networks. And the development prospects of technologies can make the overall capacity of the information system to a certain extent, to maximize the effect of $5 \mathrm{G}$ wireless network.

\subsection{Equipped With A Large Number of MIMO Technologies}

In the conventional wireless communication technology, because the number of technical antennas is limited, the data is accommodated less. But MIMO systems also have multiple antennas that connect directly to the transmitter and receiver. The number of antennas generally 
represents the capacity of the $5 \mathrm{G}$ wireless channel for data. The more the number of antennas, the more information it contains and the more data it can carry. A large number of MIMO systems are also equipped with a large number of antenna elements, which can function as a medium between the receiver and the transmitter, and it is difficult to remember whether it is a single device or a series of devices. The number of antennas can preserve the advantages of the traditional MIMO system on the one hand, and improve the application efficiency of the entire $5 \mathrm{G}$ data communication technology on the other hand, and can effectively improve the excessive noise of the conventional antenna, so that the noise will not disappear quickly. Therefore, a large number of MIMO technologies is also an alternative to $5 \mathrm{G}$ wireless networks.

\subsection{Mobile Base Station Technology}

A mobile base station mainly refers to a mobile communication base station, which is a type of station that performs mobile communication under radio coverage. Currently used mobile base stations include macro cells, micro cells, and pico cells. The Chinese name of MFemtocell technology is the femtocell, which is also a concept that has just emerged in recent years. In general, MFemtocell is an independent cell, which can absorb the core technology of the mobile network and can move back and forth, so that it can be connected through the dynamic mode in the operator's operating system, vehicles, high-speed trains, trains and other vehicles have also been widely used. The promotion of MFemtocell technology can effectively reduce the cost overhead of traditional mobile network operation, and can also improve the operating speed of mobile networks, so it can also be used as a key technology of $5 \mathrm{G}$ cellular technology. For example, only one MFemtocell can realize real-time linking and selection switching for all linked users, and can reduce a series of cumbersome steps brought by other selection switching, thereby making the network wireless communication process consume. The situation can be greatly reduced to obtain good wireless communication effects.

\subsection{Cognitive Radio Technology}

Cognitive Radio Network (CR ) is an innovative software radio technology that can improve the crowded RF spectrum of existing wireless communications and enable the radio frequency spectrum to be fully utilized most of the time. A shared frequency band of a headphone system within a CR networks can be used as a certain interference-free baseband or interference tolerance. In the CR network, it is possible to effectively perceive the surrounding radio environment, and then perform moderate adjustment of its own transmission power [3]. In an uninfected CR network, users can deploy broadband spectrum by authorizing users to use spectrum resources. The $\mathrm{CR}$ receiver first needs to monitor and allocate the spectrum resources that have not been used, and then feed the information back to the CR transmitter. The coordination mechanism mainly refers to preventing users from colliding when multiple CR networks try to access the same matching spectrum, at fault tolerant interference $\mathrm{CR}$ networks, $\mathrm{CR}$ users can share spectrum resources with the authorization system and can effectively reduce the interference threshold. By mixing the mode of the CR network of the cellular network, it is also possible to cause a further increase in bandwidth and to expand the capacity of the cellular network itself.

\subsection{Visible Light Communication}

Visible light communication technology (VLC) can use solid-state lighting LEDs as signal emitters, and then use photodiodes and avalanche photodiodes as signal receivers. This means that VLC enables the system to have a broadband radio data connection on the basis of providing illumination. In the case where illumination is not desired, this effect can also be achieved by infrared LEDs. In a VLC system, information can carry communication information by the intensity of light, while radio frequency communication uses traditional numbers of synthesized bipolar signals to make adjustments to the communication scheme. In addition, the VLC system is not affected by fast fading, and its wavelength is significantly reduced compared to the detector area. Through link-level experiments, it can be found that VLC can play a good RF communication spectrum bottleneck reduction effect, and can also pass the current mature illumination system which is used for efficient deployment of wireless optical networks, and can improve the spectrum efficiency of the network based on MU access, interference coordination and other technologies [4]. However, in the VLC system, it is also subject to a variety of external lighting factors, which requires researchers to further strengthen the research work in this area.

\subsection{Green Communication Technology}

Green communication mainly refers to energy conservation and emission reduction in the communication process as much as possible. It also needs effective protection for the surrounding ecological environment, thereby constructing a communication system with high efficiency, low consumption and recovery. The infinite communication system provided by the $5 \mathrm{G}$ wireless network also needs to fully consider the total amount of energy used in the design process, and needs to use green materials as much as possible to design, reducing the unnecessary generation during the implementation process. Waste of resources. Through the rational application of green communication technology in $5 \mathrm{G}$ communication technology, the cost expenditure in the network communication process can be greatly reduced, and the economic benefits of operators can be effectively improved. In addition, in the green communication technology, the impact of the traditional wireless network communication technology on the ecological environment can be effectively reduced, 
thereby achieving good wireless communication effects on the basis of less waste and consumption, and also reducing exhaust gas and waste emissions. Thus, the emergence of the greenhouse effect is effectively delayed [5].

\subsection{Spatial Modulation Technique}

Spatial modulation is mainly to separately encode each data, and then issue the code to a corresponding antenna position to achieve common synthesis transmission, and obtain good data transmission effect. In the $5 \mathrm{G}$ communication technology, the reasonable application of the spatial modulation technology can change the transmission mode of the traditional fixed mode, and the transmission rate can be further improved by constructing the mode of the new arrangement mode of the antenna. Although this technology seems cumbersome, in the actual operation process, a separate antenna is needed, and other antennas are mostly in the preparation stage, and can receive information or command reception at any time. In addition, through the application of spatial modulation technology, it can operate immediately after receiving the command and has a very high communication rate.

\section{Conclusion:}

5G communication technology is in the process of high-speed development in recent years. Its purpose is to enhance the wireless communication network experience of users, and let wireless communication technology better serve people's lives and work to promote the level of informationization in China. The level of digitization has been further improved. This paper mainly expounds the meaning of cellular structure and its application advantages, and then discusses the key technologies of several major $5 \mathrm{G}$ wireless communication networks in depth, hoping to give relevant communication researchers some theoretical help.

\section{References:}

1. Zhao Ge, Lei Dali. Cellular structure and key technologies of $5 \mathrm{G}$ wireless communication network [J]. Digital users, 2017, (26): 10.

2. Zhang Weiyang. On the key technologies of cellular structure and 5G wireless communication network [J]. Digital Communication World, 2018, (2): 55.

3. Wang Qiong, Qiao Kuan, Yang Zhonghao. Key Technologies of Cellular Structure and 5G Wireless Communication Network[J]. Guangdong Communication Technology, 2016, (3): 28-31, 46.

4. Sun Fansong. Wireless network resource optimization based on double-layer queue model [D]. Zhejiang University of Science and Technology, 2016.

5. Liu Tao. Key Technologies of Physical Layer of 5G Wireless Communication Network [J].
Communication World, 2017, (20): 47-48. 